\title{
Efeitos do Período e Volume de Aplicação na Segurança dos Tratoristas Aplicando Herbicidas na Cultura de CANA-De-Açúcar (Saccharum Spp.) ${ }^{1}$
}

\author{
Effects of Herbicide Spraying Period and Volume on the Safety of Tractor Drivers Spraying \\ Herbicides on Sugarcane (Saccharum spp.) Crop
}

MOMESSO, J.C. ${ }^{2}$ e MACHADO NETO, J.G. ${ }^{3}$

\begin{abstract}
RESUMO - O objetivo deste trabalho foi avaliar os efeitos do período e volume de aplicação na segurança da atividade de tratoristas aplicando herbicidas na cultura de cana-de-açúcar com o pulverizador de barra montado em trator e a eficiência de equipamentos de proteção individual (EPIs) e de uma cabina acoplada ao trator. As exposições dérmicas de 13 condições de trabalho foram avaliadas e analisadas estatisticamente por meio do delineamento inteiramente ao acaso e do esquema fatorial $3 \times 2 \times 2+1$. O fator A foi a condição de exposição: 1) exposição dérmica potencial (EDP) - sem nenhuma medida de segurança; 2) exposição com cabina no trator (Cabina); e 3) exposição com as vestimentas (EPI). O fator B foi o volume de aplicação: 1) $200 \mathrm{~L} \mathrm{ha}^{-1}$ e 2) $100 \mathrm{~L} \mathrm{ha}^{-1}$; e o fator $\mathrm{C}$ foi o período de aplicação: 1) diurno e 2) noturno. Como testemunha foi avaliada a EDP do tratorista aplicando na atividade usual de $300 \mathrm{~L}$ de calda ha ${ }^{-1}$, no periodo diurno. As exposições dérmicas (EDs) aos herbicidas considerados nessas condições de trabalho foram estimadas por meio de dados substitutos das EDs avaliadas ao cátion $\mathrm{Cu}^{+2}$ adicionado como traçador nas caldas aplicadas. O pulverizador utilizado foi do modelo PJ 600, com barra de $12 \mathrm{~m}$ de comprimento e 24 bicos de jato plano TT 11004 ou TT 110 02. As 13 condições de trabalho avaliadas foram classificadas como seguras (MS $\geq 1$ ) para o tratorista aplicando os herbicidas glyphosate (48\% i.a.), MSMA (48\%), diuron $(46,8 \%)+$ hexazinone $(13,2 \%)$, clomazone $(50 \%)$, sulfentrazone $(50 \%)$, ametryne $(50 \%)$, diuron $(50 \%)$, isoxaflutole $(75 \%)$, metribuzin $(48 \%), 2,4-\mathrm{D}(80,6 \%)$, ametryne $(30 \%)+$ clomazone $(20 \%)$, ametryne $(73,25 \%)+$ trifloxysulfuron $(1,85 \%)$ e tebuthiuron $(80 \%)$ e inseguras para o herbicida atrazine $(50 \%)$, nos dois periodos e nos três volumes de aplicação, e ametryne (50\%), na aplicação diurna e $100 \mathrm{~L} \mathrm{de} \mathrm{calda} \mathrm{ha}^{-1}$. As aplicações noturnas e os volumes de aplicação reduzidos tornaram as condições de trabalho mais seguras, exceto para o atrazine. A eficiência dos EPIs para a aplicação de $300 \mathrm{~L} \mathrm{ha}^{-1}$ variou de 69,5 a 89,3\%, e a da cabina do trator variou entre 76,4 e $83,3 \%$, em relação aos volumes reduzidos de 100 e $200 \mathrm{~L} \mathrm{ha}^{-1}$.
\end{abstract}

Palavras-chave: exposição dérmica, tratorista, herbicida, período de pulverização, volume de pulverização.

ABSTRACT - This work aimed to evaluate the effects of herbicide application period and volume on the safety of tractor drivers applying herbicide on sugar cane crops using a boom sprayer set and the efficiency of individual protective equipment (IPE), and of a cabin coupled to the tractor. Dermal exposure under thirteen working conditions was evaluated and statistically analyzed in a randomized complete design and a $3 \times 2 \times 2+1$ factorial scheme. Factor A constituted the exposure conditions: 1) potential dermal exposure (PDE) - without safety measures; 2) tractor cabin exposure (Cabin) and 3) exposure with clothes on. Factor B constituted volume applications: 1) $200 \mathrm{~L} \mathrm{ha}^{-1}$ and 2) $100 \mathrm{~L} \mathrm{ha}^{-1}$ and factor C constituted the periods of application: 1) day and 2) night. As control, the tractor driver's PDE was evaluated by applying $300 \mathrm{~L} \mathrm{ha}^{-1}$ during the day. Dermal exposure (DE) to the herbicides utilized under these working conditions was

Recebido para publicação em 10.6.2002 e na forma revisada em 12.12.2003.

2 Eng.-Agr., mestrando no Programa de Pós-Graduação em Agronomia - área de concentração em Produção Vegetal, da FCAV/ UNESP, Jaboticabal-SP, <jmomesso@ asbyte.com.br>; ${ }^{3}$ Prof. Adjunto, Dep. de Fitossanidade da FCAV/UNESP - Jaboticabal, Via de Acesso Prof. Paulo Donato Castellane, 14844-900 Jaboticabal-SP, <joaquim@fcav.unesp.br>.

Planta Daninha, Viçosa-MG, v.21, n.3, p.467-478, 2003 
calculated by substituted data of the SEs evaluated to the cation $\mathrm{Cu}^{+2}$ added to the mixture, as a tracer. The sprayer was a PJ 600 model, with $12 \mathrm{~m}$ long bar and 24 Teejet TT 110 or or TT 11002 nozzles. The thirteen working conditions evaluated were classified as safe to the tractor driver applying the herbicides glyphosate (48\% ia), MSMA (48\%), diuron (46.8\%) + hexazinone (13.2\%), clomazone (50\%), sulfentrazone (50\%), ametryne (50\%), diuron (50\%), isoxaflutole (75\%), metribuzin (48\%), 2,4 D (80.6\%), ametryne (30\%) + clomazone (20\%), ametryne $(73.25 \%)+$ trifloxysulfuron (1.85\%), tebuthiuron (80 \%) and as not safe, applying the atrazine herbicide (50\%) during the day, and $100 \mathrm{~L} \mathrm{ha}^{-1}$. Night applications and reduced application volumes provided safer work conditions, except for atrazine. IPE efficiency for the $300 \mathrm{~L} \mathrm{ha}^{-1}$ applications was around $69.5 \%$ and $89.3 \%$ and $76.4 \%$ to $83.3 \%$ for the tractor cabin, compared to the reduced volumes of 100 and $200 \mathrm{~L} \mathrm{ha}^{-1}$.

Key words: dermal exposure, tractor driver, herbicides, time application and volume application.

\section{INTRODUÇÃO}

As culturas de cana-de-açúcar são conduzidas com alto nível tecnológico, destacandose o uso de herbicidas para o controle das plantas daninhas. No ano de 2000 foram vendidas 81,86 mil toneladas de ingrediente ativo de herbicidas para a cultura de cana-de-açúcar, totalizando US\$ 142,37 milhões, correspondendo a $10,9 \%$ do total comercializado no Brasil e ao terceiro maior consumo por cultura (Sindag, 2002).

A maioria dos herbicidas utilizados na cultura de cana-de-açúcar é aplicada com pulverizadores de barra montados em trator, em que os tratoristas estão expostos e sujeitos a riscos de intoxicações ocupacionais com esses agrotóxicos. Dessa forma, há necessidade de avaliar a segurança das condições de trabalho do tratorista com esses herbicidas e, se necessário, implementar medidas de proteção.

De maneira geral, as medidas de segurança no trabalho com agrotóxicos atuam reduzindo a toxicidade ou a exposição ocupacional. A exposição ocupacional aos agrotóxicos pode ser real ou potencial. A exposição real é definida como a quantidade efetiva de produto que entra em contato com o corpo do trabalhador, podendo ser absorvida pelas vias dérmica, respiratória e oral (Bonsall, 1985). A exposição potencial é a quantidade de agrotóxico que, na ausência ou completa permeabilidade das roupas, atinge a pele do trabalhador, além das vias respiratória e oral (Turnbull et al., 1985).

A segurança das condições de trabalho com agrotóxicos pode ser determinada pela fórmula de Severn (1984), com o cálculo da margem de segurança (MS), baseada na toxicidade do agrotóxico e na exposição proporcionada pelas condições específicas de trabalho. Para as condições de trabalho classificadas como seguras (MS $\geq 1$ ) não há necessidade de se implementar medidas de segurança. Por outro lado, para aquelas classificadas como inseguras (MS< $<$ ) há necessidade de implementar medidas de segurança suficientes para torná-las seguras.

As medidas de segurança aplicadas nas condições de trabalho com os agrotóxicos podem ser classificadas em passivas e ativas. As medidas de segurança passivas atuam reduzindo apenas a exposição real, ao passo que as ativas reduzem a exposição potencial proporcionada pelas condições de trabalho e, por conseqüência, a exposição real. As medidas de segurança passivas podem ser agrupadas em individuais e coletivas, e as de segurança ativa, em apenas coletivas. As medidas de segurança individuais referem-se a cuidados com o corpo do trabalhador, enquanto as coletivas estão relacionadas com o ambiente de trabalho em que as atividades com os agrotóxicos são realizadas. Estas atuam principalmente como anteparos na trajetória do agrotóxico, entre o ponto de lançamento até antes da superfície do corpo do trabalhador. As medidas de segurança ativas coletivas são aplicadas em máquinas e equipamentos, nas condições de manejo e recomendações agronômicas e nos procedimentos operacionais, que podem contribuir para redução da exposição potencial.

De maneira geral, tem-se recomendado o uso de equipamentos de proteção individual (EPIs) como a primeira medida de segurança no trabalho com agrotóxicos. Entretanto, 
quando os trabalhadores têm acesso aos EPIs, eles são de uso insuportável em campo, pois proporcionam grande retenção de umidade e de calor na superfície do corpo (Bull \& Hathaway, 1986).

As vestimentas de proteção individual são classificadas como medida de segurança passiva individual, pois não reduzem a exposição potencial proporcionada pelas condições de trabalho com agrotóxico, diminuindo apenas a exposição real (Machado Neto, 1997). Os EPIs devem ser recomendados de acordo com a necessidade de controle da exposição, com base nos conhecimentos das exposições proporcionadas pelas condições específicas de trabalho e pelas respectivas distribuições nas partes do corpo do trabalhador (Machado Neto, 1997). Os primeiros EPIs recomendados devem ser os que protegem as partes do corpo mais expostas e em número suficiente para tornar seguras as condições de trabalho inseguras. Dessa forma, o número de EPIs recomendados geralmente é menor em relação à recomendação baseada apenas na classe toxicológica do agrotóxico, resultando em maior conforto para o usuário e menor custo para o agricultor.

As EDPs proporcionadas por diversas atividades de pulverização com agrotóxicos na cultura do algodão e a eficiência de um conjunto de proteção individual confeccionado em tecido de algodão hidrorrepelente, tratado com teflon e complementado com luvas e botas de borracha impermeáveis, foram avaliadas por Machado Neto (1997). A EDP do tratorista foi de 41,74 mL por dia aplicando com o pulverizador de barra montado em trator, e a eficiência dos EPIs, de 50,7\%.

$\mathrm{Na}$ cultura de citros foram avaliadas as EDPs dos tratoristas que aplicavam agrotóxicos com alto volume de calda (Oliveira, 2000). A EDP proporcionada ao tratorista aplicando com o pulverizador de pistolas foi de $57,2 \mathrm{~mL}$ por dia, e com o pulverizador turbopulverizador, de $217,3 \mathrm{~mL}$ por dia. A eficiência do conjunto de proteção individual, complementado com luvas e botas de borracha impermeáveis, foi de 77,8 e $96,7 \%$, para o tratorista com pulverizador de pistola e turbopulverizador, respectivamente. Ainda na cultura de citros, Lapuente (1996) verificou que o uso do conjunto de proteção complementado com luvas e botas de borracha impermeáveis reduziu 70\% a EDP do tratorista à calda de aplicação de agrotóxicos, com turbopulverizador.

A utilização de luvas de borracha na preparação da calda do herbicida DNOSBP (dinitroo-secundário de butilfenol) reduziu $75,4 \%$ a exposição das mãos (Wolfe et al., 1961). O uso de botas de borracha proporcionou controle de 95\% na exposição dérmica dos pés (Lundehn et al., 1992). Roupas de plástico, ou emborrachadas, são significativamente mais eficientes que os tecidos de algodão utilizados na proteção. Entretanto, em clima quente são muito desconfortáveis e rejeitadas pelos operários, mesmo por aqueles que estão sofrendo altas exposições (Davies et al., 1982).

As medidas de segurança passivas coletivas devem ser as primeiras a serem adotadas, como cabinas acopladas aos tratores, para proteger o tratorista da exposição aos agrotóxicos (Lapuente, 1996; Van Hemmen, 1992; Oliveira, 2000), mas ainda são pouco difundidas e utilizadas no país, quando comparadas aos EPIs. Mesmo se as condições de trabalho forem classificadas como seguras, as cabinas devem ser recomendadas como medida de segurança adicional para os tratoristas que aplicam herbicidas com o pulverizador de barra traseira em cultura de cana-de-açúcar (Momesso, 1999).

Diversos acessórios para acoplagem ao trator, com diferentes níveis de proteção ao tratorista, estão disponíveis no comércio. O acessório denominado Cabkit, composto por estrutura metálica e vidros afixados em torno da capota original do trator, reduziu $67 \%$ a EDP dos tratoristas que aplicam agrotóxicos com o pulverizador de pistolas em cultura de citros (Lapuente, 1996).

Dentre os acessórios acoplados ao trator, para proteção do tratorista, as cabinas são os mais eficientes, por serem mais fechadas que o acessório Cabkit. Cabinas das marcas Agroleite e Real controlaram, respectivamente, 85,5 e 94,9\% das EDPs dos tratoristas às caldas dos agrotóxicos, em alto volume de aplicação, com o pulverizador turbopulverizador, em citros (Oliveira, 2000).

Considerando-se ainda as medidas de segurança no trabalho com os agrotóxicos, o cálculo do tempo de trabalho seguro (TTS) pode ser utilizado como uma medida de segurança ativa de controle da exposição. Do início da 
exposição até o trabalhador receber a dose equivalente ao limite de tolerância, há um intervalo de tempo que pode ser denominado tempo de trabalho seguro (TTS). A limitação do tempo de trabalho ao TTS constitui-se em medida de segurança ativa, que pode ser aplicada a qualquer condição de trabalho e calculada com a fórmula proposta por Machado Neto (1997).

Vários fatores estão relacionados à maior ou menor exposição aos agrotóxicos, dentre os quais podem-se citar as características físicoquímicas das formulações, as condições ambientais durante a aplicação, o tempo de exposição, a manutenção de equipamentos e o uso correto de equipamentos de proteção (Goellner, 1988; Van Hemmen, 1992). Dentro do fator condições ambientais, os períodos de aplicação, diurnos e noturnos, podem influenciar a exposição dos tratoristas que aplicam herbicidas com pulverizador de barra em culturas de canade-açúcar. Além desses fatores, o volume de aplicação também pode influenciar as exposições ocupacionais aos agrotóxicos.

Volumes de pulverização menores que $300 \mathrm{~L}^{\text {de calda ha-1 }}$ são utilizados pelos agricultores como prática usual no sistema de produção agrícola. Essa prática proporciona diminuição dos reabastecimentos, dando maior autonomia aos pulverizadores, com conseqüente redução do custo de produção (Guimarães \& Valente, 1993).

Trabalhos de exposição ocupacional aos herbicidas, em períodos e volumes de aplicação reduzidos em relação aos convencionais, em cultura de cana-de-açúcar, não foram encontrados na literatura consultada.

Os objetivos deste trabalho foram: a) avaliar e comparar a EDP dos tratoristas às caldas de herbicidas com o pulverizador de barra traseira montado em trator; b) comparar as EDPs proporcionadas às aplicações diurnas e noturnas e com volumes de aplicação reduzidos (medidas de segurança ativas) com a aplicação convencional de $300 \mathrm{~L}$ de calda ha $^{-1}$ no período diurno; e c) estimar a eficiência de uma cabina no trator, como medida de segurança passiva coletiva, e de EPIs (conjunto de proteção marca AZR), como medida de segurança passiva individual, no controle das EDPs.

\section{MATERIAL E MÉTODOS}

\section{Condições de trabalho do tratorista}

As exposições dérmicas (EDs) do tratorista que aplica herbicidas na cultura de cana-deaçúcar foram quantificadas na área agrícola da Fazenda Santa Isabel, município de Jaboticabal-SP, nos meses de abril e maio de 2001. As aplicações foram realizadas com o pulverizador modelo PJ 600 de barra traseira montado em trator, em pré-emergência das plantas daninhas e no início da brotação das soqueiras de cana-de-açúcar. A barra do pulverizador tinha $12 \mathrm{~m}$ de comprimento, 24 bicos de jato plano espaçados de $0,5 \mathrm{~m}$, modelo turbo Teejet 11004 nas aplicações com volumes de 300 e $200 \mathrm{~L} \mathrm{ha}^{-1}$ e 11002 para aplicações de $100 \mathrm{~L} \mathrm{ha}^{-1}$.

Foram utilizados dois tipos de trator: um modelo MF 292, adaptado à cabina estudada, e um MF 630, apenas com a capota original de fábrica. Esses dois tratores são equivalentes em relação às dimensões e aos parâmetros operacionais. Os tratores trabalharam na velocidade de $6 \mathrm{~km}$ hora $^{-1}$ e a pressão de pulverização de 1,3 bar foi mantida constante para os volumes de 100 e $200 \mathrm{~L} \mathrm{ha}^{-1}$. Os bicos 11002 e 11004 com estes volumes de aplicação produzem gotas de diâmetro médio volumétrico de 400 a 450 micras e 500 a 550 micras, respectivamente (Spraying Systems, 1999). Como aplicação usual de campo foi considerada a pulverização com volume de $300 \mathrm{~L} \mathrm{ha}^{-1}$, no período diurno, com o trator MF 630 à velocidade de $6 \mathrm{~km}$ hora $^{-1}$ e pressão de pulverização de 2,8 bar. Nessa condição de trabalho o uso do bico 11004 produz gotas de 400 a 450 micras. As gotas produzidas são classificadas como grossas e muito grossas (Spraying Systems, 1999).

O pulverizador de barra montado em trator utilizado em todas as avaliações foi o modelo PJ 600, com tanque de 600 litros. O tempo médio de aplicação do volume de calda de um tanque foi de 54 minutos para o volume de $100 \mathrm{~L} \mathrm{ha}^{-1}, 26$ minutos para o de $200 \mathrm{~L} \mathrm{ha}^{-1} \mathrm{e}$ 20 minutos para o de $300 \mathrm{~L} \mathrm{ha}^{-1}$.

As avaliações das exposições dérmicas no período diurno ocorreram entre 8 e 16 horas e, no noturno, entre 18 e 1 hora. As avaliações das exposições potenciais (EDP), sem nenhuma 
proteção e com cabina, foram avaliadas em oito repetições, e a atividade com EPIs, em sete. As condições de trabalho estudadas e respectivas condições climáticas durante as avaliações estão apresentadas na Tabela 1. Os dados das condições de clima (Tabela 1) são médias dos valores das temperaturas, umidades relativas do ar e velocidades dos ventos durante os períodos das avaliações das exposições, que ocorrem em condições normais de trabalho dos tratoristas.

\section{Quantificação das exposições dérmicas e cálculo da eficiência das medidas de segurança testadas}

O cátion $\mathrm{Cu}^{+2}$ foi utilizado como traçador na dosagem de 300 gramas do fungicida Cuprogarb 500 para cada $100 \mathrm{~L}$ de calda. As exposições às caldas foram estimadas por meio dos dados da exposição ao cátion cobre quantificados nos amostradores das exposições e das respectivas concentrações nas caldas aplicadas. As exposições dérmicas médias foram calculadas com as exposições às caldas estimadas em cada uma das repetições. O critério de aceitação das repetições foi o seu valor estar entre a média calculada com todas as repetições e mais ou menos três vezes o desviopadrão da própria média (WHO, 1982). Os dados de exposições dérmicas às caldas que continham apenas o fungicida cúprico foram utilizados como dados substitutos para estimar as exposições aos herbicidas, de acordo com Jensen (1984). Estas exposições foram estimadas por meio das suas concentrações e quantificadas nos volumes de caldas determinados como exposições dérmicas proporcionadas pelas condições de trabalho.

Os coletores utilizados nas avaliações das exposições do corpo dos tratoristas foram macacões de tecido de algodão com capuz, luvas de algodão e absorventes higiênicos femininos (Carefree) afixados sobre máscaras semifaciais descartáveis, para quantificar a exposição na face, e na parte mediana do "peito" dos pés, para quantificar a exposição dos pés (Machado Neto \& Matuo, 1989; Machado Neto, 1990, 1997).

As exposições dérmicas das faces e dos pés foram estimadas por extrapolação da área dos absorventes $\left(70 \mathrm{~cm}^{2}\right)$ para as respectivas áreas dessas partes do corpo, de acordo com Machado Neto $(1990,1997)$. Os macacões foram seccionados em capuz, braços, pernas e tórax; para a avaliação da distribuição da exposição nas partes do corpo foram consideradas as seguintes regiões: 1) cabeça + pescoço (capuz + face); 2) mãos (luvas direita + esquerda); 3) braços (direito + esquerdo); 4) tórax - frente; 5) tórax - atrás; 6) coxas + pernas - frente (direita + esquerda); 7 ) coxas + pernas - atrás (direita + esquerda); e 8) pés.

Tabela 1 - Condições de trabalho e condições climáticas ocorridas durante as avaliações das exposições dérmicas dos tratoristas nas atividades de aplicação de herbicidas em cultura de cana-de-açúcar com o pulverizador de barra montado em trator. Jaboticabal-SP, 2002

\begin{tabular}{|c|c|c|c|c|c|c|c|c|c|c|c|c|}
\hline \multicolumn{3}{|c|}{ Condições de trabalho } & \multirow{2}{*}{\multicolumn{2}{|c|}{ Data }} & \multirow{2}{*}{\multicolumn{2}{|c|}{ Repetições }} & \multicolumn{6}{|c|}{ Condições de tempo } \\
\hline Proteção & Período & $\begin{array}{l}\text { Volume } \\
\left(\mathrm{L} \mathrm{ha}^{-1}\right)\end{array}$ & & & & & \multicolumn{2}{|c|}{$\begin{array}{c}\text { Temperatura } \\
\left({ }^{\circ} \mathrm{C}\right)\end{array}$} & \multicolumn{2}{|c|}{$\begin{array}{l}\text { UR do ar } \\
(\%)\end{array}$} & \multicolumn{2}{|c|}{$\begin{array}{l}\text { Vel. do Vento } \\
\left(\mathrm{km} \mathrm{h}^{-1}\right)\end{array}$} \\
\hline 1. Sem proteção & Diurno & 200 & \multicolumn{2}{|c|}{$24 / 04 / 2002$} & \multicolumn{2}{|c|}{1 a 8} & \multicolumn{2}{|c|}{34,1} & \multicolumn{2}{|c|}{40,6} & \multicolumn{2}{|c|}{4,9} \\
\hline 2. Sem proteção & Diurno & 100 & $25 / 04$ & $26 / 04$ & 1 a 6 & 7 e 8 & 34,3 & 25,5 & 36,5 & 52,8 & 7,7 & 3,6 \\
\hline 3. Sem proteção & Noturno & 200 & \multicolumn{2}{|c|}{$30 / 04$} & \multicolumn{2}{|c|}{1 a 8} & \multicolumn{2}{|c|}{22,4} & \multicolumn{2}{|c|}{59,1} & \multicolumn{2}{|c|}{3,4} \\
\hline 4. Sem proteção & Noturno & 100 & $26 / 04$ & $27 / 04$ & 1 a 4 & 5 a 8 & 20,8 & 22,8 & 65,2 & 72,4 & 3,9 & 3,1 \\
\hline 5. Sem proteção & 'Diurno & 300 & \multicolumn{2}{|c|}{$10 / 05$} & \multicolumn{2}{|c|}{1 a 8} & \multicolumn{2}{|c|}{31,5} & \multicolumn{2}{|c|}{40,0} & \multicolumn{2}{|c|}{5,1} \\
\hline 6. Cabina & Diurno & 200 & \multicolumn{2}{|c|}{$24 / 04$} & \multicolumn{2}{|c|}{1 a 8} & \multicolumn{2}{|c|}{34,1} & \multicolumn{2}{|c|}{40,6} & \multicolumn{2}{|c|}{4,9} \\
\hline 7. Cabina & Diurno & 100 & $25 / 04$ & $26 / 04$ & 1 a 6 & 7 e 8 & 34,3 & 25,5 & 36,5 & 52,8 & 7,7 & 3,6 \\
\hline 8. Cabina & Noturno & 200 & \multicolumn{2}{|c|}{$30 / 04$} & \multicolumn{2}{|c|}{1 a 8} & \multicolumn{2}{|c|}{22,4} & \multicolumn{2}{|c|}{59,1} & \multicolumn{2}{|c|}{3,4} \\
\hline 9. Cabina & Noturno & 100 & $26 / 04$ & $27 / 04$ & 1 a 4 & 5 a 8 & 20,8 & 22,8 & 65,2 & 72,4 & 3,9 & 3,1 \\
\hline 10. EPI * & 'Diurno & 200 & \multicolumn{2}{|c|}{$03 / 05 / 02$} & \multicolumn{2}{|c|}{1 a 8} & \multicolumn{2}{|c|}{35,1} & & & & \\
\hline 11. EPI & Diurno & 100 & $03 / 05$ & $10 / 05$ & 1 a 5 & 6 a 8 & 35,1 & 31,5 & 36,2 & 40,0 & 4,2 & 5,1 \\
\hline 12. EPI & Noturno & 200 & & & & & & & & & & \\
\hline 13. EPI & Noturno & 100 & $07 / 05$ & $08 / 05$ & 1 a 4 & 5 a 8 & 16,6 & 18,4 & 66,1 & 63,3 & 4,3 & 4,0 \\
\hline
\end{tabular}

* EPI - equipamento de proteção individual (conjunto marca AZR). 
As medidas de segurança avaliadas para o controle da exposição do tratorista estão apresentadas na Tabela 1 . As medidas de segurança ativas foram: 1) período de aplicação noturno para comparação com o diurno; e 2) reduções do volume de aplicação de $300 \mathrm{~L}$ de calda ha ${ }^{-1}$ para 200 e $100 \mathrm{~L} \mathrm{ha}^{-1}$. As medidas de segurança passivas foram: 1) coletiva: uso de cabina de proteção ao tratorista e 2) individual: equipamentos de proteção individual. Foi utilizada a cabina da marca Real, modelo 12.581, dotada de um climatizador de ar, que proporciona aumento da umidade relativa e diminuição da temperatura do ar interno. Os equipamentos de proteção individual foram compostos pelo conjunto de proteção da marca AZR, que consistia em uma calça comprida com cordel na cintura; camisa de mangas compridas com gola em $\mathrm{V}$, fechada com velcro, que também tinha o cordel na região da cintura; e toca "árabe" com aba frontal, tipo boné, e fechada com velcro na região abaixo do queixo. Todas as peças desse conjunto AZR foram confeccionadas em tecido com 100\% de algodão e hidrorrepelente, devido ao tratamento com Teflon $\AA$. Também fizeram parte desse conjunto AZR uma viseira transparente de acetato e um par de luvas de borracha nitrílica.

As partes seccionadas dos macacões e os coletores foram retirados dos trabalhadores imediatamente após os períodos de exposição e acondicionados em sacos plásticos identificados. Esses coletores foram levados ao laboratório para a solubilização do cátion $\mathrm{Cu}^{+2}$, com solução de $\mathrm{HCl}$ a $0,1 \mathrm{~N}$, de acordo com o procedimento desenvolvido por Machado Neto \& Matuo (1989). As concentrações do traçador nos extratos foram quantificadas em espectrofotômetro de absorção atômica.

A eficiência dos EPIs foi calculada por meio da diferença entre a EDP, obtida no trator com capota original de fábrica, e a exposição dérmica não controlada (EDNC), avaliada nas vestimentas amostradoras usadas sob EPIs avaliados, exceto para a máscara e bota de borracha, cujas eficiências foram consideradas como de 90 e 95\%, respectivamente (Lundehn et al., 1992; Kieczka, 1993). A eficiência da cabina foi calculada por meio da diferença entre a EDP do tratorista avaliada com apenas a capota original do trator e a EDP com a cabina em avaliação.
As exposições dérmicas avaliadas nas 13 condições de trabalho foram analisadas estatisticamente por meio do delineamento inteiramente ao acaso e no esquema fatorial $3 \times 2 \times 2$ +1 . O fator A foi a condição de trabalho: 1) exposição dérmica potencial (EDP) - sem nenhuma medida de segurança; 2) exposição com cabina no trator (Cabina); e 3) exposição com os equipamentos de proteção individual (EPI). O fator B foi o volume de aplicação: $200 \mathrm{~L} \mathrm{ha}^{-1}$ e $100 \mathrm{~L} \mathrm{ha}^{-1}$; e o fator $\mathrm{C}$ foi o período de aplicação: diurno e noturno. Como testemunha, foi avaliada a EDP do tratorista que aplicava, na forma usual, $300 \mathrm{~L}$ de calda $\mathrm{ha}^{-1}$ no período diurno.

\section{Cálculo da segurança das condições de trabalho}

A segurança das condições de trabalho do tratorista com os herbicidas listados na Tabela 2 foi calculada com a fórmula da margem de segurança (MS) proposta por Severn (1984) e adaptada por Machado Neto (1997):

$$
\mathrm{MS}=\frac{\text { NOEL } \times 70}{\text { QAE } \times 10}
$$

Nessa fórmula, o NOEL é o Nível de Efeito Não Observável publicado por Worthing \& Hance (1991) ou fornecido pelo fabricante (Tabela 2). Esse valor, expresso em $\mathrm{mg} \mathrm{kg}^{-1} \mathrm{dia}^{-1}$, é obtido em testes de avaliação da toxicidade crônica em animais em laboratório, sendo um dado toxicológico obrigatório para o registro dos agrotóxicos. O teste para determinação do NOEL é feito com a administração oral de doses crescentes dos ingredientes ativos dos agrotóxicos, nas dietas dos animais. O número 70 é o peso corpóreo considerado para o trabalhador (kg). A QAE é a Quantidade Absorvivel das Exposições avaliadas nas vias dérmicas e respiratórias em cada condição de trabalho (mg dia ${ }^{-1}$ ). A QAE foi calculada considerandose a absorção dos herbicidas na via dérmica como $10 \%$ da exposição dérmica avaliada (Smith, 1984). A absorção na via respiratória foi considerada como $1 \%$ da exposição dérmica avaliada, pois normalmente representa menos de $1 \%$ da exposição total (Van Hemmen, 1992). O número 10 multiplicando a $\mathrm{QAE}$ é um fator de segurança utilizado para compensar a extrapolação dos dados toxicológicos obtidos em 
animais de laboratório para o homem (Brouwer et al., 1990).

O critério utilizado para classificar a segurança das condições de trabalho em função do valor MS, de acordo com Machado Neto (1997), foi o seguinte: Se MS $\geq 1$, a condição é segura, a exposição é aceitável e o risco de intoxicação é tolerável, pois a quantidade absorvivel da exposição multiplicada pelo fator de segurança 10 é menor que a quantidade absorvivel segura (NOEL x $70 \mathrm{~kg}$ ). Se MS < 1, a condição é insegura, a exposição é intolerável e o risco de intoxicação é inaceitável, visto que o denominador da fórmula ( $\mathrm{QAE} \times 10$ ) é maior que a quantidade absorvivel segura.

\section{Cálculo do tempo de trabalho seguro (TTS)}

O TTS foi estimado com a fórmula proposta por Machado Neto (1997):

\section{TTS $=$ MS $x$ tee}

Nessa fórmula, MS é a margem de segurança estimada e tee é o tempo de exposição

Tabela 2 - Herbicidas recomendados para o controle das plantas daninhas na cultura de cana-de-açúcar e considerados neste estudo de segurança do tratorista, aplicandoos com o pulverizador de barra montado em trator. Jaboticabal-SP, 2002

\begin{tabular}{|c|c|c|c|}
\hline \multicolumn{3}{|c|}{ Herbicida } & \multirow[b]{2}{*}{$\begin{array}{c}\text { NOEL** } \\
\left(\mathrm{mg} \mathrm{kg}^{-1} \mathrm{dia}^{-1}\right)\end{array}$} \\
\hline Nome comum & \begin{tabular}{|c|} 
Concentração \\
ingrediente ativo \\
$(\%)$
\end{tabular} & $\mid \begin{array}{c}\text { Dose } * \\
\left(\mathrm{~L} \mathrm{ou} \mathrm{kg} \mathrm{ha}^{-1}\right)\end{array}$ & \\
\hline glyphosate & 48,00 & $5,0-7,0$ & 31,0 \\
\hline MSMA & 48,00 & $4,0-6,0$ & 6,0 \\
\hline diuron + & 46,80 & \multirow{2}{*}{$1,8-3,0$} & 12,5 \\
\hline hexazinone & 13,20 & & 10,0 \\
\hline clomazone & 50,00 & $2,0-3,0$ & 4,3 \\
\hline sulfentrazone & 50,00 & $1,2-2,0$ & 10,0 \\
\hline atrazine & 50,00 & $4,0-8,0$ & 0,5 \\
\hline ametryne & 50,00 & $3,0-6,0$ & 2,5 \\
\hline diuron & 50,00 & $3,0-6,0$ & 12,5 \\
\hline isoxaflutole & 75,00 & $1,0-1,6$ & 2,0 \\
\hline metribuzin & 48,00 & 1,0 & 10,0 \\
\hline 2,4-D & 80,60 & $2,5-3,8$ & 62,5 \\
\hline ametryne + & 30,00 & \multirow{2}{*}{$5,0-6,0$} & 2,5 \\
\hline clomazone & 20,00 & & 4,3 \\
\hline ametryne & 73,25 & \multirow{2}{*}{$1,50-2,0$} & 2,5 \\
\hline trifloxysulfuron & 1,85 & & 15,0 \\
\hline tebuthiuron & 80,00 & $0,6-1,5$ & 40,0 \\
\hline
\end{tabular}

* Compêndio (1999).

** NOEL - Nível de efeito não observável - Worthing \& Hance (1991) efetiva por dia de trabalho, considerado em seis horas. Se MS $\geq 1$, o TTS será maior que o tee e se confirma a intensidade da segurança da condição de trabalho em análise. Se MS < 1, o TTS será menor que o tee e o seu cálculo possibilita a restrição do tempo de exposição diária ao TTS, utilizando-o como uma medida de segurança ativa.

\section{RESULTADOS E DISCUSSÃO}

\section{Exposições dérmicas potenciais às caldas e estimativas da eficiência das medidas de segurança testadas}

As exposições dérmicas potenciais (EDPs) e as exposições dérmicas não controlada (EDNCs) pelos EPIs e pela cabina do trator às caldas contendo o fungicida Cuprogarb 500, proporcionadas pelas 13 atividades avaliadas, estão apresentadas na Tabela 3. A maior ED proporcionada ao tratorista ocorreu na aplicação de $300 \mathrm{~L}_{\text {de calda ha }} \mathrm{h}^{-1}$ e no periodo diurno - atividade considerada usual de campo.

Na Tabela 3 verifica-se que, em relação à atividade convencional, as reduções do volume e o período de aplicação proporcionaram controles de 27, 1 a $61,7 \%$ das EDPs recebidas pelo tratorista. O uso da cabina no trator proporcionou reduções na EDP do tratorista entre 38,5 e $77,0 \%$ e, com o uso dos EPIs, entre 49,2 e $85,2 \%$, independentemente da redução de volume e do período de aplicação. Observouse também que os volumes e períodos de aplicação testados, associados ao uso de cabina no trator, proporcionaram reduções entre 76,4 e $83,3 \%$ das EDPs ao tratorista e, com o uso do conjunto de proteção, entre 69,5 e $89,3 \%$, em relação à condição usual de trabalho (aplicação de $300 \mathrm{~L} \mathrm{ha}^{-1}$ no período diurno).

Pode-se constatar na Tabela 4 que as condições de trabalho que compuseram o fatorial, o volume e os períodos de aplicação proporcionaram exposições dérmicas ao tratorista significativamente inferiores à condição usual de trabalho, resultando, na média, em $67,2 \%$ de controle (contraste fatorial $\mathrm{x}$ testemunha). Embora não diferindo significativamente, verifica-se uma tendência de menores exposições dérmicas do tratorista nas aplicações com volume de $100 \mathrm{~L} \mathrm{ha}^{-1}$, em relação a $200 \mathrm{~L} \mathrm{ha}^{-1}$ (21,7\% menor), e do período noturno em 
Tabela 3 - Valores médios das exposições dérmicas potenciais (EDPs) e das exposições dérmicas não controladas (EDNC) proporcionadas ao tratorista nas atividades de aplicação de herbicidas em cultura de cana-de-açúcar com o pulverizador de barra montado em trator. Jaboticabal-SP, 2002

\begin{tabular}{|c|c|c|c|c|}
\hline \multirow{2}{*}{ Atividade } & \multicolumn{2}{|c|}{ EDPs } & \multicolumn{2}{|c|}{ EDNC - \% Controle } \\
\hline & $\mathrm{mL} \mathrm{dia}^{-1}$ & D. P. * & / EDP & / $300 \mathrm{~L} \mathrm{ha}^{-1}$ \\
\hline EDP $-200 \mathrm{~L} \mathrm{ha}^{-1}-$ Diurno & 7,20 & 3,96 & - & 40,1 \\
\hline $\mathrm{EDP}-100 \mathrm{~L} \mathrm{ha}^{-1}-$ Diurno & 8,76 & 1,51 & - & 27,1 \\
\hline $\mathrm{EDP}-200 \mathrm{~L} \mathrm{ha}^{-1}-$ Noturno & 8,01 & 1,51 & - & 33,4 \\
\hline EDP - $100 \mathrm{~L} \mathrm{ha}^{-1}-$ Noturno & 4,60 & 1,17 & - & 61,7 \\
\hline EDP $-300 \mathrm{~L} \mathrm{ha}^{-1}-$ Diurno & 12,02 & 9,60 & - & - \\
\hline Cabina - 200 L ha ${ }^{-1}$ - Diurno & 2,37 & 0,68 & 67,1 & 80,3 \\
\hline Cabina - $100 \mathrm{~L} \mathrm{ha}^{-1}-$ Diurno & 2,01 & 0,51 & 77,0 & 83,3 \\
\hline Cabina - 200 L ha ${ }^{-1}$ - Noturno & 2,42 & 1,12 & 69,8 & 79,9 \\
\hline Cabina - $100 \mathrm{~L} \mathrm{ha}^{-1}$ - Noturno & 2,83 & 2,06 & 38,5 & 76,4 \\
\hline EPI - $200 \mathrm{~L} \mathrm{ha}^{-1}-$ Diurno & 3,66 & 1,71 & 49,2 & 69,5 \\
\hline EPI - $100 \mathrm{~L} \mathrm{ha}^{-1}$ - Diurno & 1,30 & 0,67 & 85,2 & 89,2 \\
\hline EPI - 200 L ha ${ }^{-1}-$ Noturno & 2,86 & 1,32 & 64,3 & 76,2 \\
\hline EPI - $100 \mathrm{~L} \mathrm{ha}^{-1}-$ Noturno & 1,28 & 1,06 & 72,2 & 89,3 \\
\hline
\end{tabular}

* D.P. $=$ desvio-padrão da média.

relação ao diurno (13\% menor). Observa-se também nesta tabela que a EDP do tratorista foi significativamente controlada pelas medidas de segurança passivas testadas. Em média, a cabina reduziu a EDP do tratorista em $66,2 \%$, e os EPIs, em 68,2\%. Esses resultados estão de acordo com os obtidos por outros autores quando avaliaram a eficiência de cabines e equipamentos de proteção individual em pulverizações nas culturas de citros (Lapuente, 1996; Oliveira, 2000) e de algodão (Machado Neto, 1997).

O coeficiente de variação de 70,19\% obtido na análise fatorial (Tabela 4) é perfeitamente aceitável, uma vez que os dados médios de exposição dérmica das repetições nas atividades estudadas não excederam os limites de três vezes o desvio-padrão, conforme o protocolo da Organização Mundial de Saúde (WHO, 1982).

\section{Exposição dérmica aos herbicidas, margem de segurança, necessidade de controle da exposição e tempo de trabalho seguro}

As estimativas das EDPs e EDNCs aos herbicidas, as margens de segurança (MSs) e os tempos de trabalho seguro (TTSs) calculados para o tratorista nas 13 atividades avaliadas estão apresentados nas Tabelas 5 a
9. Para esses cálculos foram consideradas as maiores doses recomendadas dos herbicidas (Compêndio, 1999). A atividade convencional (aplicação de $300 \mathrm{~L}$ de calda ha $^{-1}$ no período diurno) foi classificada como segura (MS $\geq 1$ ) para o tratorista nos cálculos para as aplicações de todos os herbicidas considerados (Tabela 5), exceto para atrazine, única condição de trabalho classificada como insegura (MS $<1$ ). Os valores das MSs calculados resultaram em TTSs proporcionalmente superiores ao período considerado de seis horas de jornada diária de trabalho. Para a atividade insegura com o herbicida atrazine, o TTS calculado foi de apenas $1,18 \mathrm{~h}$, valor muito baixo e não adequado para ser considerado como medida de segurança (Tabela 5). Esse resultado ocorreu porque o NOEL da atrazine é baixo $\left(0,5 \mathrm{mg} \mathrm{kg}^{-1} \mathrm{dia}^{-1}\right)$, influenciando diretamente o cálculo da MS. Nesses dados verifica-se que herbicidas mais tóxicos, com menores valores de NOEL, para a mesma condição de uso e dose, podem proporcionar exposição ocupacional insegura ao trabalhador $(\mathrm{MS}<1)$ e viceversa. Por outro lado, para o herbicida 2,4 - D, cujo NOEL é de 62,5 $\mathrm{mg} \mathrm{kg}^{-1} \mathrm{dia}^{-1}$ (Tabela 3), a margem de segurança calculada foi de 123,04. Esses resultados refletem o efeito da toxicidade dos herbicidas na segurança das condições de trabalho. 
Tabela 4 - Resultados das análises estatísticas dos dados de exposições dérmicas às caldas proporcionadas ao tratorista nas atividades de aplicação de herbicidas na cultura de cana-de-açúcar com o pulverizador de barra montado em trator. Jaboticabal-SP, 2002

\begin{tabular}{|c|c|}
\hline Causas de variação & Valores de F \\
\hline Proteção $(\mathrm{P})$ & $24,00 * *$ \\
\hline Volume de aplicação (VA) & $2,14 \mathrm{~ns}$ \\
\hline Período de aplicação (PA) & $0,71 \mathrm{~ns}$ \\
\hline $\mathrm{PxVA}$ & $0,78 \mathrm{~ns}$ \\
\hline $\mathrm{P} \times \mathrm{PA}$ & $0,88 \mathrm{~ns}$ \\
\hline $\mathrm{VA} \times \mathrm{PA}$ & $0,77 \mathrm{~ns}$ \\
\hline $\mathrm{P} \times \mathrm{VA} \times \mathrm{PA}$ & $2,14 \mathrm{~ns}$ \\
\hline Tratamentos & $5,38 * *$ \\
\hline Tratamentos $\mathrm{x}$ testemunha & $46,98 * *$ \\
\hline $\mathrm{CV}(\%)$ & 70,19 \\
\hline Condição de trabalho & $\mathrm{mL} \mathrm{dia}^{-1}$ \\
\hline EDP & $7,14 \mathrm{a}^{1^{\prime}}$ \\
\hline Cabina & $2,41 \mathrm{~b}$ \\
\hline EPI & $2,27 \mathrm{~b}$ \\
\hline DMS $(5 \%)$ & 1,91 \\
\hline \multicolumn{2}{|l|}{ Volume de pulverização } \\
\hline $200 \mathrm{~L} \mathrm{ha}^{-1}$ & $4,42 \mathrm{a}$ \\
\hline $100 \mathrm{~L} \mathrm{ha}^{-1}$ & $3,46 \mathrm{a}$ \\
\hline DMS $(5 \%)$ & 1,30 \\
\hline \multicolumn{2}{|l|}{ Período de aplicação } \\
\hline Dia & $4,22 \mathrm{a}$ \\
\hline Noite & $3,67 \mathrm{a}$ \\
\hline $\operatorname{DMS}(5 \%)$ & 1,30 \\
\hline \multicolumn{2}{|l|}{ Testemunha $x$ Fatorial } \\
\hline Testemunha & $12,02 \mathrm{a}$ \\
\hline Fatorial & $3,94 \mathrm{~b}$ \\
\hline $\operatorname{DMS}(5 \%)$ & 1,30 \\
\hline
\end{tabular}

*** e ns - significativo a $1 \%, 5 \%$ e não-significativo, respectivamente.

${ }^{1 /}$ Médias seguidas da mesma letra na coluna e no mesmo fator não diferem entre si pelo teste de Tukey $(\mathrm{P}>0,05)$.

Na aplicação no período diurno com volume de $200 \mathrm{~L}$ de calda ha ${ }^{-1}$ (Tabela 6), todas as atividades consideradas foram classificadas como seguras, exceto para atrazine, classificada como insegura, mesmo com o uso das medidas de segurança passivas testadas. Para a aplicação com o volume de $100 \mathrm{~L}$ de calda ha ${ }^{-1}$ (Tabela 7), cuja EDP foi 21,7\% menor que a da aplicação com $200 \mathrm{~L} \mathrm{ha}^{-1}$ (Tabela 4), também foi classificada como insegura a aplicação de atrazine, inclusive com o uso das medidas de segurança passiva e do herbicida ametryne, que se tornou segura com o uso da cabina do trator e dos EPIs. As demais aplicações nessa condição de trabalho foram classificadas como seguras (Tabela 7).

Tabela 5 - Valores médios das exposições dérmicas potenciais (EDP), margem de segurança (MS) e tempo de trabalho seguro (TTS) do tratorista nas atividades de aplicação dos herbicidas com o pulverizador de barra montado em trator, no período diurno e com o volume de $300 \mathrm{~L}^{\text {de calda }} \mathrm{ha}^{-1}$. Jaboticabal-SP, 2002

\begin{tabular}{|c|c|c|c|c|}
\hline \multicolumn{2}{|c|}{ Herbicida } & \multirow{2}{*}{$\begin{array}{c}\text { EDP } \\
\left(\mathrm{mg} \mathrm{dia}^{-1}\right)\end{array}$} & \multirow{2}{*}{ MS } & \multirow{2}{*}{ TTS (h) } \\
\hline Nome comum & $\%$ p.c & & & \\
\hline glyphosate & 2,4 & 138,47 & 14,25 & 85,48 \\
\hline MSMA & 2,0 & 115,39 & 3,31 & 19,85 \\
\hline diuron + & \multirow{2}{*}{1,0} & 56,25 & 14,14 & 84,84 \\
\hline hexazinone & & 15,87 & 40,11 & 240,65 \\
\hline clomazone & 1,0 & 60,10 & 4,55 & 27,32 \\
\hline sulfentrazone & 0,7 & 42,07 & 15,13 & 90,76 \\
\hline atrazine & 2,7 & 162,27 & 0,20 & 1,18 \\
\hline ametryne & 2,0 & 120,20 & 1,32 & 7,94 \\
\hline diuron & 2,0 & 120,20 & 6,62 & 39,71 \\
\hline isoxafrutole & 0,5 & 47,78 & 2,66 & 15,98 \\
\hline metribuzin & 0,35 & 20,19 & 31,51 & 189,08 \\
\hline $2,4-\mathrm{D}$ & 1,27 & 123,04 & 32,33 & 193,95 \\
\hline ametryne + & \multirow{2}{*}{2,0} & 72,12 & 2,21 & 13,24 \\
\hline clomazone & & 48,08 & 5,69 & 34,15 \\
\hline ametryne + & \multirow{2}{*}{0,67} & 58,99 & 2,70 & 16,18 \\
\hline trifloxisulfuron & & 1,49 & 640,69 & $3.844,12$ \\
\hline tebuthiuron & 0,5 & 138,47 & 14,25 & 85,48 \\
\hline
\end{tabular}

p.c. - produto comercial na calda.

Nas aplicações no período noturno, com os volumes de 100 e $200 \mathrm{~L}$ de calda ha ${ }^{-1}$ (Tabelas 8 e 9), todas as atividades consideradas foram classificadas como seguras, exceto com atrazine, classificada como insegura, mesmo com o uso das medidas de segurança passivas testadas.

Considerando esses resultados, verifica-se que formulações com atrazine devem ser preteridas durante o processo de recomendação, pois a aplicação deste herbicida foi classificada como insegura para o tratorista em todas as atividades avaliadas, inclusive com o uso das medidas de segurança passivas testadas. Para os demais herbicidas considerados (Tabela 3), cujas condições de trabalho foram classificadas como seguras, as medidas de segurança passivas (cabina e EPIs) até poderiam ser dispensadas, porém deveriam ser recomendadas como medidas preventivas de exposições acidentais. 
Tabela 6 - Valores médios das exposições dérmicas, margem de segurança (MS) e tempo de trabalho seguro (TTS) do tratorista nas atividades de aplicação dos herbicidas com o pulverizador de barra montado em trator, no período diurno e com o volume de $200 \mathrm{~L}$ de calda ha ${ }^{-1}$. Jaboticabal-SP, 2002

\begin{tabular}{|c|c|c|c|c|c|c|c|c|c|c|}
\hline \multicolumn{2}{|c|}{ Herbicida } & \multicolumn{3}{|c|}{$\begin{array}{c}\text { Exposições Dérmicas } \\
\left(\mathrm{mg} \mathrm{dia}^{-1}\right)\end{array}$} & \multicolumn{3}{|c|}{ MS } & \multicolumn{3}{|c|}{ TTS (h) } \\
\hline Nome comum & $\begin{array}{c}\% \\
\text { p.c }\end{array}$ & EDP* & EPI** & $\mathrm{CAB}^{* * * *}$ & EDP & EPI & $\mathrm{CAB}$ & EDP & EPI & $\mathrm{CAB}$ \\
\hline glyphosate & 3,5 & 120,96 & 53,76 & 39,82 & 16,31 & 36,70 & 49,55 & 97,85 & 220,17 & 297,28 \\
\hline MSMA & 3,0 & 103,68 & 46,08 & 34,13 & 3,68 & 8,29 & 11,19 & 22,10 & 49,72 & 67,13 \\
\hline diuron + & \multirow{2}{*}{1,5} & 50,54 & 22,46 & 16,64 & 15,74 & 35,41 & 47,81 & 94,43 & 212,46 & 286,87 \\
\hline hexazinone & & 14,26 & 6,34 & 4,69 & 44,64 & 100,44 & 135,61 & 267,83 & 602,62 & 813,66 \\
\hline clomazone & 1,5 & 54,00 & 24,00 & 17,78 & 5,07 & 11,40 & 15,39 & 30,40 & 68,41 & 92,37 \\
\hline sulfentrazone & 1,0 & 36,00 & 16,00 & 11,85 & 17,68 & 39,77 & 53,70 & 106,06 & 238,64 & 322,21 \\
\hline atrazine & 4,0 & 144,00 & 64,00 & 47,40 & 0,22 & 0,50 & 0,67 & 1,33 & 2,98 & 4,03 \\
\hline ametryne & 3,0 & 108,00 & 48,00 & 35,55 & 1,47 & 3,31 & 4,48 & 8,84 & 19,89 & 26,85 \\
\hline diuron & 3,0 & 108,00 & 48,00 & 35,55 & 7,37 & 16,57 & 22,38 & 44,19 & 99,43 & 134,25 \\
\hline isoxafrutole & 0,8 & 43,20 & 19,20 & 14,22 & 2,95 & 6,63 & 8,95 & 17,68 & 39,77 & 53,70 \\
\hline metribuzin & 0,5 & 17,28 & 7,68 & 5,69 & 36,83 & 82,86 & 111,88 & 220,96 & 497,16 & 671,27 \\
\hline $2,4-\mathrm{D}$ & 2,0 & 116,06 & 51,58 & 38,20 & 34,27 & 77,10 & 104,11 & 205,61 & 462,62 & 624,63 \\
\hline ametryne + & \multirow{2}{*}{3,0} & 64,80 & 28,80 & 21,33 & 2,46 & 5,52 & 7,46 & 14,73 & 33,14 & 44,75 \\
\hline clomazone & & 43,20 & 19,20 & 14,22 & 6,33 & 14,25 & 19,24 & 38,01 & 85,51 & 115,46 \\
\hline ametryne + & \multirow{2}{*}{1,0} & 52,74 & 23,44 & 17,36 & 3,02 & 6,79 & 9,16 & 18,10 & 40,72 & 54,98 \\
\hline trifloxysulfuron & & 1,33 & 0,59 & 0,44 & 716,63 & $1.612,40$ & $2.177,00$ & $4.299,70$ & $9.674,40$ & $13.062,00$ \\
\hline tebuthiuron & 0,8 & 46,08 & 20,48 & 15,17 & 55,24 & 124,29 & 167,82 & 331,44 & 745,74 & $1.006,90$ \\
\hline
\end{tabular}

p.c. - produto comercial na calda. * EDP - exposição dérmica potencial - sem proteção. ** EPI - equipamento de proteção individual (conjunto AZR). *** CAB - cabina.

Tabela 7 - Valores médios das exposições dérmicas, margem de segurança (MS) e tempo de trabalho seguro (TTS) do tratorista nas atividades de aplicação dos herbicidas com o pulverizador de barra montado em trator, no período diurno e com o volume de $100 \mathrm{~L}_{\text {de calda ha }}{ }^{-1}$. Jaboticabal-SP, 2002

\begin{tabular}{|c|c|c|c|c|c|c|c|c|c|c|}
\hline \multicolumn{2}{|c|}{ Herbicida } & \multicolumn{3}{|c|}{$\begin{array}{c}\text { Exposições Dérmicas } \\
\left(\mathrm{mg} \mathrm{dia}^{-1}\right)\end{array}$} & \multicolumn{3}{|c|}{ MS } & \multicolumn{3}{|c|}{ TTS (h) } \\
\hline Nome comum & $\begin{array}{l}\% \\
\text { p.c }\end{array}$ & EDP* & EPI** & $\mathrm{CAB} * * *$ & EDP & EPI & CAB & EDP & EPI & $\mathrm{CAB}$ \\
\hline glyphosate & 7,0 & 294,34 & 43,68 & 67,54 & 6,70 & 45,16 & 29,21 & 40,21 & 270,98 & 175,26 \\
\hline MSMA & 6,0 & 252,29 & 37,44 & 57,89 & 1,51 & 10,20 & 6,60 & 9,08 & 61,19 & 39,57 \\
\hline diuron + & \multirow{2}{*}{3,0} & 122,99 & 18,25 & 28,22 & 6,47 & 43,58 & 28,19 & 38,81 & 261,49 & 169,12 \\
\hline hexazinone & & 34,69 & 5,15 & 7,96 & 18,34 & 123,61 & 79,95 & 110,07 & 741,68 & 479,70 \\
\hline clomazone' & 3,0 & 131,40 & 19,50 & 30,15 & 2,08 & 14,03 & 9,08 & 12,49 & 84,20 & 54,45 \\
\hline sulfentrazone & 2,0 & 87,60 & 13,00 & 20,10 & 7,26 & 48,95 & 31,66 & 43,59 & 293,71 & 189,96 \\
\hline atrazine & 8,0 & 350,40 & 52,00 & 80,40 & 0,09 & 0,61 & 0,40 & 0,54 & 3,67 & 2,37 \\
\hline ametryne & 6,0 & 262,80 & 39,00 & 60,30 & 0,61 & 4,08 & 2,64 & 3,63 & 24,48 & 15,83 \\
\hline diuron & 6,0 & 262,80 & 39,00 & 60,30 & 3,03 & 20,40 & 13,19 & 18,16 & 122,38 & 79,15 \\
\hline isoxafrutole & 1,6 & 9,86 & 1,46 & 2,26 & 12,91 & 87,02 & 56,28 & 77,49 & 522,14 & 337,71 \\
\hline metribuzin & 1,0 & 67,28 & 9,98 & 15,44 & 9,46 & 63,74 & 41,22 & 56,75 & 382,43 & 247,34 \\
\hline 2,4-D & 4,0 & 282,42 & 41,91 & 64,80 & 14,08 & "94,90" & 61,38 & 84,50 & 569,37 & 368,25 \\
\hline ametryne + & \multirow{2}{*}{6,0} & 157,68 & 23,40 & 36,18 & 1,01 & 6,80 & 4,40 & 6,05 & 40,79 & 26,38 \\
\hline clomazone' & & 105,12 & 15,60 & 24,12 & 2,60 & 17,54 & 11,34 & 15,62 & 105,24 & 68,07 \\
\hline ametryne + & \multirow{2}{*}{2,0} & 128,33 & 19,05 & 29,45 & 1,24 & 8,35 & 5,40 & 7,44 & 50,12 & 32,42 \\
\hline trifloxysulfuron & & 3,24 & 0,48 & 0,74 & 294,50 & $1.984,5$ & $1.283,5$ & $1.767,00$ & $11.907,00$ & $7.701,00$ \\
\hline tebuthiuron & 1,5 & 56,06 & 8,32 & 12,86 & 45,40 & 305,94 & 197,87 & 272,42 & $1.835,60$ & $1.187,20$ \\
\hline
\end{tabular}

p.c. - produto comercial na calda. * EDP - exposição dérmica potencial - sem proteção. ** EPI - equipamento de proteção individual (conjunto AZR). *** CAB - cabina.

Planta Daninha, Viçosa-MG, v.21, n.3, p.467-478, 2003 
Tabela 8 - Valores médios das exposições dérmicas, margem de segurança (MS) e tempo de trabalho seguro (TTS) do tratorista nas atividades de aplicação dos herbicidas com o pulverizador de barra montado em trator, no período noturno e com o volume de $200 \mathrm{~L}_{\text {de calda }} \mathrm{ha}^{-1}$. Jaboticabal-SP, 2002

\begin{tabular}{|c|c|c|c|c|c|c|c|c|c|c|}
\hline \multicolumn{2}{|l|}{ Herbicida } & \multicolumn{3}{|c|}{$\begin{array}{c}\text { Exposições Dérmicas } \\
\left(\mathrm{mg} \mathrm{dia}^{-1}\right)\end{array}$} & \multicolumn{3}{|c|}{ MS } & \multicolumn{3}{|c|}{ TTS (h) } \\
\hline Nome comum & $\begin{array}{c}\% \\
\text { p.c }\end{array}$ & $\mathrm{EDP}^{*}$ & $\mathrm{EPI}^{* *}$ & $\mathrm{CAB} * * *$ & EDP & EPI & $\mathrm{CAB}$ & EDP & EPI & $\mathrm{CAB}$ \\
\hline glyphosate & 3,5 & 134,57 & 48,05 & 40,66 & 14,66 & 41,06 & 48,52 & 87,96 & 246,34 & 291,13 \\
\hline MSMA & 3,0 & 115,34 & 41,18 & 34,85 & 3,31 & 9,27 & 10,96 & 19,86 & 55,63 & 65,74 \\
\hline diuron + & \multirow{2}{*}{1,5} & 56,23 & 20,08 & 16,99 & 14,15 & 39,62 & 46,82 & 84,88 & 237,72 & 280,94 \\
\hline hexazinone & & 15,86 & 5,66 & 4,79 & 40,12 & 112,38 & 132,81 & 240,75 & 674,26 & 796,85 \\
\hline clomazone & 1,5 & 60,08 & 21,45 & 18,15 & 4,55 & 12,76 & 15,08 & 27,33 & 76,54 & 90,46 \\
\hline sulfentrazone & 1,0 & 40,05 & 14,30 & 12,10 & 15,89 & 44,50 & 52,59 & 95,34 & 267,01 & 315,55 \\
\hline atrazine & 4,0 & 160,20 & 57,20 & 48,40 & 0,20 & 0,56 & 0,66 & 1,19 & 3,34 & 3,94 \\
\hline ametryne & 3,0 & 120,15 & 42,90 & 36,30 & 1,32 & 3,71 & 4,38 & 7,94 & 22,25 & 26,30 \\
\hline diuron & 3,0 & 120,15 & 42,90 & 36,30 & 6,62 & 18,54 & 21,91 & 39,72 & 111,25 & 131,48 \\
\hline isoxafrutole & 0,8 & 48,06 & 17,16 & 14,52 & 2,65 & 7,42 & 8,77 & 15,89 & 44,50 & 52,59 \\
\hline metribuzin & 0,5 & 19,22 & 6,86 & 5,81 & 33,10 & 92,71 & 109,57 & 198,62 & 556,26 & 657,40 \\
\hline $2,4-D$ & 2,0 & 129,12 & 46,10 & 39,01 & 30,80 & 86,27 & 101,95 & 184,82 & 517,61 & 611,72 \\
\hline ametryne + & \multirow{2}{*}{3,0} & 72,09 & 25,74 & 21,78 & 2,21 & 6,18 & 7,30 & 13,24 & 37,08 & $\begin{array}{l}43,83 \\
4\end{array}$ \\
\hline clomazone & & 48,06 & 17,16 & 14,52 & 5,69 & 15,95 & 18,85 & 34,16 & 95,68 & 113,07 \\
\hline ametryne + & \multirow{2}{*}{1,0} & 58,67 & 20,95 & 17,73 & 2,71 & 7,59 & 8,97 & 16,27 & 45,56 & 53,85 \\
\hline trifloxysulfuron & & 1,48 & 0,53 & 0,45 & 644,16 & $1.804,10$ & $2.132,10$ & $\begin{array}{l}3.864,90 \\
3 .\end{array}$ & $10.824,00$ & $12.792,00$ \\
\hline tebuthiuron & 0,8 & 51,26 & 18,30 & 15,49 & 49,65 & 139,07 & 164,35 & 297,92 & 834,39 & 986,10 \\
\hline
\end{tabular}

p.c. - produto comercial na calda. * EDP - exposição dérmica potencial - sem proteção. ** EPI - equipamento de proteção individual (conjunto AZR). *** $\mathrm{CAB}-$ cabina.

Tabela 9 - Valores médios das exposições dérmicas, margem de segurança (MS) e tempo de trabalho seguro (TTS) do tratorista nas atividades de aplicação dos herbicidas com o pulverizador de barra montado em trator, no período noturno e com o volume de $100 \mathrm{~L}_{\text {de calda }} \mathrm{ha}^{-1}$. Jaboticabal-SP, 2002

\begin{tabular}{|c|c|c|c|c|c|c|c|c|c|c|}
\hline \multicolumn{2}{|l|}{ Herbicida } & \multicolumn{3}{|c|}{$\begin{array}{c}\text { Exposições Dérmicas } \\
\left(\mathrm{mg} \mathrm{dia}^{-1}\right)\end{array}$} & \multicolumn{3}{|c|}{ MS } & \multicolumn{3}{|c|}{ TTS (h) } \\
\hline Nome comum & $\begin{array}{l}\% \\
\text { p.c }\end{array}$ & EDP* & $\mathrm{EPI}^{* *}$ & $\mathrm{CAB} * * *$ & EDP & EPI & $\mathrm{CAB}$ & EDP & EPI & $\mathrm{CAB}$ \\
\hline glyphosate & 7,0 & 154,56 & 43,01 & 95,09 & 12,76 & 45,87 & 20,75 & 76,58 & 275,21 & 124,48 \\
\hline MSMA & 6,0 & 132,48 & 36,86 & 81,50 & 2,88 & 10,36 & 4,68 & 17,29 & 62,14 & 28,11 \\
\hline diuron + & \multirow{2}{*}{3,0} & 64,58 & 17,97 & 39,73 & 12,32 & 44,26 & 20,02 & 73,90 & 265,58 & 120,12 \\
\hline hexazinone & & 18,22 & 5,07 & 11,21 & 34,93 & 125,55 & 56,78 & 209,61 & 753,27 & 340,70 \\
\hline clomazone & 3,0 & 69,00 & 19,20 & 42,45 & 3,97 & 14,25 & 6,45 & 23,79 & 85,51 & 38,68 \\
\hline sulfentrazone & 2,0 & 46,00 & 12,80 & 28,30 & 13,83 & 49,72 & 22,49 & 83,00 & 298,30 & 134,92 \\
\hline atrazine & 8,0 & 184,00 & 51,20 & 113,2 & 0,17 & 0,62 & 0,28 & 1,04 & 3,73 & 1,69 \\
\hline ametryne & 6,0 & 138,00 & 38,40 & 84,90 & 1,15 & 4,14 & 1,87 & 6,92 & 24,86 & 11,24 \\
\hline diuron & 6,0 & 138,00 & 38,40 & 84,90 & $\begin{array}{l}5,76 \\
5\end{array}$ & 20,71 & 9,37 & 34,58 & 124,29 & 56,22 \\
\hline isoxafrutole & 1,6 & 55,20 & 15,36 & 33,96 & 2,31 & 8,29 & 3,75 & 13,83 & 49,72 & 22,49 \\
\hline metribuzin & 1,0 & 22,08 & 6,14 & 13,58 & 28,82 & 103,57 & 46,85 & 172,92 & 621,45 & 281,08 \\
\hline $2,4-\mathrm{D}$ & 4,0 & 148,30 & 41,27 & 91,24 & 26,82 & 96,38 & 43,59 & 160,91 & 578,27 & 261,55 \\
\hline ametryne + & \multirow{2}{*}{6,0} & 82,80 & 23,04 & 50,94 & 1,92 & 6,90 & 3,12 & 11,53 & 41,43 & 18,74 \\
\hline clomazone & & 55,20 & 15,36 & 33,96 & 4,96 & 17,81 & 8,06 & 29,74 & 106,89 & 48,35 \\
\hline ametryne + & \multirow{2}{*}{2,0} & 67,39 & 18,75 & 41,46 & 2,36 & 8,48 & 3,84 & 14,16 & 50,90 & 23,02 \\
\hline trifloxysulfuron & & 1,70 & 0,47 & 1,05 & 560,84 & $2.015,50$ & 911,61 & $3.365,00$ & $12.093,00$ & $5.469,60$ \\
\hline tebuthiuron & 1,5 & 29,44 & 8,19 & 18,11 & 86,46 & 310,72 & 140,54 & 518,77 & $1.864,30$ & 843,24 \\
\hline
\end{tabular}

p.c. - produto comercial na calda. * EDP - exposição dérmica potencial - sem proteção. ** EPI - equipamento de proteção individual (conjunto AZR). *** $\mathrm{CAB}$ - cabina. 


\section{LITERATURA CITADA}

BONSALL, J. L. Measurement of occupational exposure to pesticide. In: TURNBULL, G. I. Occupational hazards of pesticide use. London: Taylor e Francis, 1985. p. 13-33.

BROUWER, D. H. et al. Respiratory exposure to fieldstrength dusts in greenhouses during application and after re-entry. Delph, TNO Health Research, 1990. p. 183-184. (Annual Report).

BULL, D.; HATHAWAY, D. Pragas e venenos: agrotóxicos no Brasil e no terceiro mundo. Petrópolis, Vozes, 1986. $235 \mathrm{p}$.

COMPÊNDIO de defensivos agrícolas: guia prático de produtos fitossanitários para uso agrícola. 6.ed. São Paulo: Organização Andrei, 1999. 672 p.

DAVIES, J. E. et al. Protective clothing studies in the field: an alternative to reentry. In: PLIMMER, J. R. Pesticide residues and exposure. Washington: ACS, 1982. p. 170182.

GUIMARÃES, S. C.; VALENTE, T. O. Bicos de baixa vazão no controle de desmódio com chlorimuron-ethyl na cultura da soja. In: CONGRESSO BRASILEIRO DE HERBICIDAS E PLANTAS DANINHAS, 19., 1993, Londrina. Resumos... Londrina: 1993. p. 267.

GOELLNER, C. Equipamentos de proteção individual no caso de manipulação dos defensivos agrícolas. Passo Fundo: Universidade de Passo Fundo: 1988. 70 p. (Séries em Toxicologia - Boletim Técnico, v. 1, n. 2)

JENSEN, J. K. The assumptions used for exposure assessments. In: SIEWIERSKI, M. (Ed.) Determination and assessment of pesticide exposure. New York: Elsevier, 1984. p. $147-152$.

KIECZKA, H. Requierements for safe-guarding the health of applicators of plant protection products - an overview. In: INTERNATIONAL SYMPOSIUM ON PESTICIDES APPLICATION TECHNIQUES, 2., 1993, Strasbourg. Annales... Strasbourg: v. 2/2. p. 455-462.

LAPUENTE, D. B. G. Segurança das condições de aplicação de agrotóxicos na cultura do cítrus (Citrus sinensis (L.) Osbeck). 1996. 47 f. (Trabalho apresentado para graduação em Agronomia) - Universidade Estadual de São Paulo, Jaboticabal, 1996.

LUNDEHN. J. et al. Uniform principles for safeguarding the health of applicators of plant protection products (Uniform principles for operator protection). Berlin: Kommissionsverlag Paul Parey, 1992. 90 p.

MACHADO NETO, J. G. Quantificação e controle da exposição dérmica de aplicadores de agrotóxicos na cultura estaqueada de tomate, na região de Cravinhos, SP. 1990. 112 f. Tese (Doutorado em Agronomia) Universidade Estadual Paulista, Jaboticabal, 1990.
MACHADO NETO, J. G. Estimativas do tempo de trabalho seguro e da necessidade de controle da exposição ocupacional dos aplicadores de agrotóxicos. 1997. 83 f. Tese (Livre Docência em Agronomia) Universidade Estadual Paulista, Jaboticabal, 1997.

MACHADO NETO, J. G.; MATUO, T. Avaliação de um amostrador para o estudo da exposição dérmica potencial de aplicadores de defensivos agrícolas. Ci. Agron.

Jaboticabal, v. 4, n. 2, p. 21-22, 1989.

MOMESSO, J. C. Gerenciamento das condições do ambiente de trabalho das pessoas envolvidas com aplicação de herbicidas na cultura da cana-de-açúcar. 1999. 83 f. (Monografia apresentada para obtenção de título de Engenheiro de Segurança do Trabalho) Instituição Moura Lacerda, Ribeirão Preto, 1999.

OLIVEIRA, M. L. Segurança no trabalho de aplicação de agrotóxicos com turbo-atomizador e pulverizador de pistolas em cítrus. 2000. $100 \mathrm{f}$. Tese (Mestrado em Agronomia - Produção Vegetal) - Universidade Estadual Paulista, Jaboticabal, 2000.

SEVERN, D. J. Use of exposure data for risk assessment. In: SIEWIERSKI, M. (Ed.) Determination and assessment of pesticide exposure. New York: Elsevier, 1984. p. 13-19. (Studies in Environment Science, 24).

SINDAG. Consumo de defensivos agrícolas no Brasil. Disponível em: <http//www.sindag.com.br/db/arqs $>$. Acesso em: 10/2/2002.

SMITH, L. W. Development of safe use practices for pesticides. In: SIEWIERSKI, M. (Ed.) Determination and assessment of pesticide exposure. New York: Elsevier, 1984. p. 39-52.

SPRAYNG SYSTEMS Co. Produtos para pulverização na agricultura. Wheaton: North Avenue at Schmale Road, 1999. $104 \mathrm{p}$

TURNBULL, G. L.; SANDERSON, D. M.; CROME, S. J. Exposure to pesticide during application. In: Occupational hazards of pesticide use. London: Taylor \& Francis, 1985. p. 35-49.

Van HEMMEN, J. J. Agricultural pesticide exposure data bases for risk assessment. Rev. Environ. Contam. Toxicol. v. 126 , p. $1-85,1992$

WOLFE, H. R.; DURHAN, W. F.; DATCHELOR, G. S. Health hazards of some dinitro compounds. Arch. Environ. Health, v. 3, p. 468-475, 1961.

WORLD HEALTH ORGANIZATION - WHO. Field surveys of exposure to pesticide - standard protocol. Geneva: WHO, 1982. (Document VBC/82.1)

WORTHING, C. R.; HANCE, R. J. The pesticide manual, a world compendium. 9.ed. London: The British Crop Protection Council, 1991. 1141 p. 